\title{
Editorial: Rational Design and Characterization of Innovative Multifunctional Biomimetic Materials
}

\author{
Andrea Cochis ${ }^{1 *}$, Lia Rimondini ${ }^{1 *}$ and Lorenzo Moroni ${ }^{2 *}$ \\ ${ }^{1}$ Department of Health Sciences, Center for Translational Research on Autoimmune and Allergic Diseases CAAD, Universitá del \\ Piemonte Orientale UPO, Novara, Italy, ${ }^{2}$ Complex Tissue Regeneration Department, MERLN Institute for Technology-Inspired \\ Regenerative Medicine, Maastricht University, Maastricht, Netherlands
}

Keywords: biomaterials, biomimetic, multifunctional, tissue engineering, personalized medicine

Editorial on the Research Topic

Rational Design and Characterization of Innovative Multifunctional Biomimetic Materials

OPEN ACCESS

Edited by:

Nihal Engin Vrana,

Sparta Medical, France

Reviewed by:

Amir M. Ghaemmaghami, University of Nottingham,

United Kingdom

*Correspondence:

Andrea Cochis

andrea.cochis@med.uniupo.it

Lia Rimondini

lia.rimondini@med.uniupo.it Lorenzo Moroni

I.moroni@maastrichtuniversity.nI

Specialty section:

This article was submitted to Biomaterials,

a section of the journal

Frontiers in Materials

Received: 20 November 2020 Accepted: 06 January 2021

Published: 10 March 2021

Citation:

Cochis A, Rimondini $L$ and Moroni $L$ (2021) Editorial: Rational Design and

Characterization of Innovative Multifunctional Biomimetic Materials.

Front. Mater. 8:631664.

doi: 10.3389/fmats.2021.631664
Bone tissue engineering has been the most frequent topic as it was addressed by five articles dealing with different aspects with such a challenging aim. In their work entitled "In vivo Regeneration of Mineralized Bone Tissue in Anisotropic Biomimetic Sponges," Serrano-Bello et al. demonstrated the pivotal role of the device architecture for a successful repair; accordingly, alginate 3D scaffolds with anisotropic microporous domains enriched with hydroxyapatite (HA) demonstrated a high osteogenic potency toward dental pulp mesenchymal stem cells. These scaffolds were also successful in the repair of a bone defect in vivo realized in a rat calvaria model. Along with scaffold composition improvement, in their article "Enhancement of the Biological and Mechanical Performances of Sintered Hydroxyapatite by Multiple Ions Doping," Sprio et al. demonstrated the importance of ions in ameliorating biological properties. In particular, the addition of $\mathrm{Mg}^{2+}, \mathrm{Sr}^{2+}$, and $\mathrm{Zn}^{2+}$ ions to $\mathrm{HA}$ composites was crucial in influencing the phase composition and microstructure of HA/ $\beta$ TCP composites formed during the sintering process. Moreover, the ions promoted osteogenesis and inhibited bacterial infection, thus demonstrating strong improvement in comparison with the bulk HA. Similarly, de Melo Pereira et al. demonstrated the role of calcium phosphate mineral in successfully mimicking a bone-like matrix in their article "Proliferation and Osteogenic Differentiation of hMSCs on Biomineralized Collagen." Here, biomineralized collagen with intrafibrillar calcium phosphate mineral was compared to the bulk collagen in terms of proangiogenic potency toward mesenchymal stromal cells; the results demonstrated that biomineralization improved osteogenic gene expression with emphasis toward osteoprotegerin (OPG) due to its involvement in osteoclastogenesis inhibition. Culture conditions and the cell source for bone substitutes were investigated by Salgado et al. in their work "Clarifying the ToothDerived Stem Cells Behavior in a 3D Biomimetic Scaffold for Bone Tissue Engineering Applications." Accordingly, mesenchymal stem/stromal cells were isolated from human dental follicles and pulp tissues, showing high propensity to be differentiated into bone-like phenotypes when cultured on a 3D porous scaffold made of collagen-nanohydroxyapatite/phosphoserine cryogel; moreover, dynamic culture was compared to static culture, showing a different outcome in terms of alkaline phosphatase (ALP) and osteopontin (OPN), and also, the use of dental follicle stem cells reported better tissue ingrowth and osteogenic differentiation. Hence, the choice of the cell source and culture condition was crucial in influencing bone maturation. Finally, the possibility to exploit biomaterials as drug delivery systems has been investigated for the treatment of osteosarcoma by Dewhurst et al. in their work "Development of Natural-Based Bone Cement for a Controlled 
Doxorubicin-Drug Release.” PLGA nanoparticles loaded with doxorubicin (DOXO) were mixed with calcium phosphate-based bone cement in order to improve the DOXO release in the tumor site, thus limiting the drug side effect on nontarget sites. This targeting strategy was successful in ameliorating the DOXO release that was observed as particularly effective in reducing the viability of cancer stem cells, cells responsible for metastasis progression and drug resistance headway. Dealing with joint function recovery in patients with end-stage hip and knee arthritis, Couto et al. reported an interesting update about periprosthetic inflammation due to wear debris in the review article "The Mechanisms Underlying the Biological Response to Wear Debris in Periprosthetic Inflammation." The presence of such debris can be the trigger of an acute inflammation, eliciting the activation of osteoclasts and consequent periprosthetic osteolysis (PPOL), ultimately leading to implant aseptic loosening, which is the most common cause of long-term implant failure. Therefore, understanding the cellular and the molecular mechanisms underlying the biological response to implant debris can open to novel therapeutic strategies preventing PPOL.

Hydrogels have also been frequently cited in this special issue due to their tunable properties supporting the healing of different tissues. Neves et al. offered a critical revision of the improvement required by alginate hydrogels in order to ameliorate their mechanical and biological properties in the review article "Modulating Alginate Hydrogels for Improved Biological Performance as Cellular 3D Microenvironments.” By fine-tuning different properties, such as the molecular weight, backbone block composition and distribution, polymer concentration, and type/amount of cross-linkers, it is possible to modulate the viscoelastic properties, thus ameliorating the mechanical properties; the use of cell instructive/responsive peptides guiding adhesion (RGD) and degradation can instead influence cell behavior, thus making alginate hydrogels a bioactive 4D dynamic microenvironment. Hydrogels were also shown to be able to mimic a tumor microenvironment to study the metastatic progression, as described by Balion et al. in "Investigation of Cancer Cell Migration and Proliferation on Synthetic Extracellular Matrix Peptide Hydrogels." Here, the relation between the ECM and the tumorigenic cell migration and proliferation was investigated due to the hydrogel composition: so, synthetic hydrogel matrices from multi-arm polyethylene glycol (PEG) functionalized with collagen-like peptide (CLP) CG(PKG)4(POG)4(DOG)4 alone and conjugated with either cell adhesion peptide RGD (mimicking fibronectin) or IKVAV (mimicking laminin) were considered. The results showed that neither RGD nor IKVAV conjugated to PEG-CLP stimulated migratory capacity of melanoma cells, thus giving highlights regarding ECM signaling in cancer progression.

The use of cold atmospheric plasma jet (CAPJ) was treated by Lou et al. in their article "Helium/Argon-Generated Cold Atmospheric Plasma Facilitates Cutaneous Wound Healing" to improve wound healing. Thanks to an accurate regulation of the CAPJ-generated gas helium $(\mathrm{He})$ - or gas mixture of $\mathrm{He}$ and argon (He/Ar), it was possible to improve the keratinocyte proliferation and migration through the activation of epithelial-to-mesenchymal transition (EMT) and cell cycle progression, which was evidenced by a decrease in E-cadherin levels. As a consequence, in vivo experiments confirmed that the CAPJ treatment facilitated granulation tissue formation and mitigated inflammation in the cutaneous tissue, resulting in accelerated wound closure in a rat model.

Graphene has been renamed as the "miracle material" due to its exceptional mechanical properties. In this special issue, a very interesting article dealing with the use of graphene oxide (GO) to realize superior bioactive materials comes from Majkowska et al. "Interfacial Self-Assembly to Spatially Organize Graphene Oxide Into Hierarchical and Bioactive Structures." The authors described the use of supramolecular co-assembly between an elastin-like recombinamer (ELR5) and a peptide amphiphile (PA) to organize GO flakes into bioactive structures across multiple scales. The process takes advantage of a reaction-diffusion-mechanism to enable the incorporation and spatial organization of GO within multiple ELR5/PA layers. Such technical procedures open the possibility to obtain biocompatible ELR5/PA/GO membranes.

Biomaterials aimed for clinical application are very promising sources to improve the practice. Vascular grafts were realized for dialysis in the article proposed by Wu et al. "Optimization of Antikinking Designs for Vascular Grafts Based on Supramolecular Materials," where the authors aimed at developing anti-kinking properties for synthetic vascular grafts to be applied as access grafts for hemodialysis. Supramolecular materials based on hydrogen bonding interactions between bis-urea (BU) or 2-ureido-4[1H]pyrimidinones (UPy) were combined to produce microporous anti-kinking tubular structures by combining solution electrospinning with $3 \mathrm{D}$ printing. Two different tubular grafts were developed: one tubular scaffold composed of BU-polycarbonate electrospun layers with 3D-printed polycaprolactone (PCL) and the other graft fully consisted of supramolecular polymers, using chain-extended UPy-PCL as electrospun layers and a bifunctional UPy-PCL. Both grafts demonstrated a reproducible dimensional stability and antikinking behavior under bending stresses.

Finally, the most original article of this special issue comes probably from Contessi Negrini et al. "Plant Tissues as 3D Natural Scaffolds for Adipose, Bone and Tendon Tissue Regeneration," where the authors open the possibility to convert plant tissues (i.e., apple, carrot, and celery) into tissuedecellularized scaffolds coming from vegetables. Thanks to their different mechanical properties and natural fibers orientation, the vegetable scaffolds were able to support cells adhesion, alignment, and osteogenic differentiation. These results suggest that besides the well-known animal source, vegetables represent a new front for decellularized matrices in tissue engineering.

Taken together, these innovative articles fully comply with the broad scope of the special issue to report an overview of novel strategies to improve the field of biomaterials and tissue engineering, thus giving the opportunity to the readers to learn new solutions to keep in mind for their future studies. In our vision, the rationale design of a multifunctional biomaterial should consider and combine the following 
properties: 1) pro-regenerative stimulation, 2) tissue-mimicking mechanical resistance and architecture, and 3) antibacterial activity. A common underestimation is probably to consider such properties as single aloof characteristics, whereas it is more and more evident that the implant success depends on each of the abovementioned parameters. So, besides the large literature regarding the exploitability of pro-regenerative biochemical factors, we are now better understanding that other parameters such as the mechanical stimulation and the device tissue-specific architecture were probably underestimated as they represent key factors for the tissue repair as well.

In parallel with the biomaterial design, more efforts should be fueled into improvement of in vitro models to study the device performances. Using 3D models, bioreactors, and organ-on-achip for mimicking the naive tissue structure and the crosstalk between neighbor tissues are of crucial importance to better simulate a physiological environment, thus better predict biomaterials success.

Finally, we would like to focus on the importance of introducing antibacterial properties as the rapid increase in drug-resistant strains is a primary reason for infections in the implant tissue, leading to implant failure, despite the design and performances of the applied devices. This is still an underestimated problem, so it is of a crucial importance to study and verify the use of innovative antibacterial tools in order to reduce the infection rate of implantable biomaterials.

\section{AUTHOR CONTRIBUTIONS}

All authors listed have made a substantial, direct, and intellectual contribution to the work and approved it for publication.

\section{ACKNOWLEDGMENTS}

The continuous advancement in desgning and using multifunctional biomimetic materials in biomedical engineering applications spurred the interest to initiate this special issue collection. The special issue Rational Design and Characterization of Innovative Multifunctional Biomimetic Materials is now closed, so we would like to take this opportunity to sincerely thanks all the Authors for their exceptional contributions. 12 Manuscripts have been published thanks to the efforts of 89 Authors covering different topics and aspects related to the advances of biomimetic and multifunctional biomaterials.

Conflict of Interest: The authors declare that the research was conducted in the absence of any commercial or financial relationships that could be construed as a potential conflict of interest.

Copyright (c) 2021 Cochis, Rimondini and Moroni. This is an open-access article distributed under the terms of the Creative Commons Attribution License (CC BY). The use, distribution or reproduction in other forums is permitted, provided the original author(s) and the copyright owner(s) are credited and that the original publication in this journal is cited, in accordance with accepted academic practice. No use, distribution or reproduction is permitted which does not comply with these terms. 